\title{
Clinical Performance of Radiofrequency Ablation for Treatment of Uterine Fibroids: Systematic Review and Meta-Analysis of Prospective Studies
}

\author{
Linda D. Bradley, MD, ${ }^{1}$ Resad P. Pasic MD, PhD, ${ }^{2}$ and Larry E. Miller, $\mathrm{PhD}^{3}$
}

\begin{abstract}
Background: Radiofrequency ablation (RFA) has emerged as a safe and effective treatment option for women with symptomatic uterine fibroids and can be delivered by laparoscopic, transvaginal, or transcervical approaches. The evidence regarding typical patient outcomes with RFA has not previously been examined in a comprehensive fashion.

Materials and Methods: We performed a systematic review of prospective studies for treatment of uterine fibroids with RFA. Main outcomes were procedure time, patient recovery metrics, change in fibroid volume, symptom severity score (SSS), health-related quality of life (HRQL), and reinterventions. Data were analyzed with random effects meta-analysis and metaregression.

Results: We identified 32 articles of 1283 unique patients (median age: 42 years) treated with laparoscopic RFA (19 articles), transvaginal RFA (8 articles), or transcervical fibroid ablation (5 articles). Mean procedure time was 49 minutes, time to discharge was 8.2 hours, time to normal activities was 5.2 days, and time to return to work was 5.1 days. At 12 months follow-up, fibroid volume decreased by 66\%, HRQL increased by 39 points, and SSS decreased by 42 points (all $P<.001$ versus baseline). The annual cumulative rate of reinterventions due to fibroid-related symptoms was $4.2 \%, 8.2 \%$, and $11.5 \%$ through 3 years.

Conclusions: RFA of uterine fibroids significantly reduces fibroid volume, provides significant durable improvements in fibroid-related quality of life, and is associated with favorable reintervention rates.
\end{abstract}

Keywords: laparoscopic, leiomyoma, myoma, radiofrequency, transcervical, transvaginal

\section{Introduction}

$\mathbf{U}$ TERINE FIBROIDS are the most common benign solid pelvic tumor in women, developing in $\sim 70 \%$ to $80 \%$ of women by 50 years of age. ${ }^{1}$ More than 1 in 3 women with uterine fibroids report symptoms that interfere with activities of daily living such as heavy menstrual bleeding and/or bulk symptoms. ${ }^{2}$ Self-management with nonprescription medication or lifestyle modification is common, but often unsuccessful. $^{3}$ Several surgical and interventional treatments are available to women with persistent symptoms attributable to uterine fibroids, including hysterectomy, myomectomy, and uterine artery embolization. However, patient acceptance of these treatments may be limited due to the increasing demand for less invasive therapies that preserve the uterus. ${ }^{3}$

Radiofrequency ablation (RFA) has emerged as a safe and effective treatment alternative as the procedure can be delivered in a minimally invasive fashion. RFA may be delivered by a laparoscopic, transvaginal, or transcervical approach into the uterine fibroid to induce coagulative necrosis $^{4}$ with subsequent reduction in fibroid-related symptoms. Previous reviews, often limited to a single device or treatment route, have reported patient outcomes following

${ }^{1}$ Department of Obstetrics and Gynecology, Women's Health Institute, Center for Menstrual Disoders, Cleveland Clinic, Cleveland, Ohio.

${ }^{2}$ Department of Obstetrics and Gynecology, University of Louisville Hospital, Louisville, Kentucky.

${ }^{3}$ Miller Scientific Consulting, Inc., Asheville, North Carolina.

(c) Linda D. Bradley et al. 2020; Published by Mary Ann Liebert, Inc. This Open Access article is distributed under the terms of the Creative Commons License (http://creativecommons.org/licenses/by/4.0), which permits unrestricted use, distribution, and reproduction in any medium, provided the original work is properly cited. 
laparoscopic RFA., ${ }^{5,6}$ To the authors' knowledge, no systematic review has evaluated the clinical utility of each RFA delivery approach for the treatment of uterine fibroids. We hypothesized that RFA would provide significant decreases in fibroid volume and improvements in quality of life for women with symptomatic uterine fibroids. The primary aim of this study was to report the effectiveness of RFA for symptomatic uterine fibroids by means of a systematic review and meta-analysis.

\section{Materials and Methods}

\section{Eligibility criteria and search strategy}

The conduct, analysis, and reporting of this systematic review adhered to the Preferred Reporting Items for Systematic Reviews and Meta-analyses (PRISMA). ${ }^{7}$ Prospective studies of RFA for symptomatic uterine fibroid treatment were eligible for inclusion in this systematic review. We considered randomized trials, comparative cohort studies, and noncomparative cohort studies for this review, and extracted data only from the RFA arms of the study. We excluded case reports and studies with less than 10 patients, studies in which patients received concomitant surgeries due to a potential for confounding of patient outcomes, and studies that reported no main outcomes. No date or language restrictions were applied to the searches. We performed systematic searches of Medline, Embase, and the Cochrane Central Register of Controlled Trials for potentially eligible studies. Additional searches were conducted in the Directory of Open Access Journals and Google Scholar. Manual searches of the reference lists of included articles and relevant meta-analyses were performed. The search strategy included combinations of anatomic-, diagnosis-, and treatment-specific keywords. The Medline search strategy is provided in Supplementary Table S1; the search strategy for other databases was adapted as necessary.

Two researchers with expertise in systematic reviews independently screened titles and abstracts for eligibility. Full-text articles were obtained for all potentially relevant studies. To account for multiple articles derived from the same primary study or subsamples of the primary study, we preferentially extracted data from the article reporting the longest follow-up duration on the entire cohort and supplemented any missing data using other articles derived from that study. Thus, all reported data were derived from unique patients. Disagreements related to study eligibility were resolved by discussion and consensus. The final searches were performed on May 31, 2019.

\section{Data extraction}

Researchers independently extracted data from eligible studies using standardized data collection forms. For each study, we recorded metadata, patient characteristics, study characteristics, treatment regimens, and main outcomes. Main outcomes included procedure time, length of stay, time to normal activities, time to return to work, change in uterine fibroid volume, change in symptom severity score (SSS) and health-related quality of life (HRQL) on the Uterine Fibroid Symptom Health-Related Quality of Life Questionnaire (UFS-QoL), ${ }^{8}$ and surgical reinterventions.

We extracted fibroid volume, SSS, and HRQL data at baseline, 3 months, 6 months, 12 months, and beyond 12 months, where the last interval consisted of the latest follow-up interval beyond 12 months reported in each study. The rate of surgical reinterventions for fibroid-related symptoms was calculated at 6 months and annually thereafter through 3 years. Reinterventions were conservatively assumed to be performed for fibroid-related symptoms unless explicitly stated otherwise in the article. We used the National Institute of Health assessment tool for before/after studies to evaluate the methodological quality of eligible studies. ${ }^{9}$ Data extraction discrepancies between the two researchers were resolved by discussion and consensus.

\section{Statistical analyses}

Procedure time, length of stay, time to normal activities, and time to return to work were reported using the weighted mean statistic. Change in uterine fibroid volume was reported as a weighted percent change from baseline. Change in SSS and HRQL was reported using the weighted mean difference relative to baseline. The surgical reintervention rate was reported as a weighted event rate. Outcome estimates were calculated for each study and the overall pooled result was reported along with the $95 \%$ confidence interval (CI). We prospectively specified an inverse variance random effects model for all analyses given the variation among study designs and methods of RFA delivery. We evaluated temporal trends in fibroid volume, UFS-QoL scores, and reinterventions by pooling data at distinct follow-up intervals.

We estimated heterogeneity among studies with the $I^{2}$ statistic, where a value of $0 \%$ represented no heterogeneity and larger values represented increasing heterogeneity. ${ }^{10}$ In accordance with Cochrane Collaboration recommendations, we performed metaregression analysis for any outcome reported in at least 10 studies. We tested the robustness of the meta-analysis conclusions with three sensitivity analysis, including a reanalysis using a fixed-effects meta-analysis model, a one-study removed analysis where the metaanalysis was recalculated following iterative one-at-a-time removal of each study, and reanalysis of only the studies with the highest methodological quality. $P$-values were two sided with a significance level <.05. Analyses were performed using Stata v14.2 (StataCorp).

\section{Results}

\section{Study selection}

We identified 505 articles in our searches and ultimately included 32 articles of 1283 unique patients treated with RFA for uterine fibroids in this systematic review. A PRISMA flow diagram depicting the study identification and selection process is provided in Figure 1. Among the full-text articles that were reviewed, 51 were excluded, with review articles (25), case reports (7), and non-RFA treatments (6) the most common reasons (Supplementary Table S2).

\section{Patient and study characteristics}

Baseline patient characteristics in each study are reported in Table 1. Among included studies, mean patient age ranged from 39 to 45 years (median 42 years), the number of treated fibroids ranged from 1 to 5 (median 1.7) per patient, and fibroid volume ranged from 10 to $305 \mathrm{~cm}^{3}$ (median $74 \mathrm{~cm}^{3}$ ). Baseline UFS-QoL scores ranged from 22 to 77 for HRQL (median 49) and 32 to 76 for SSS (median 55). Study 


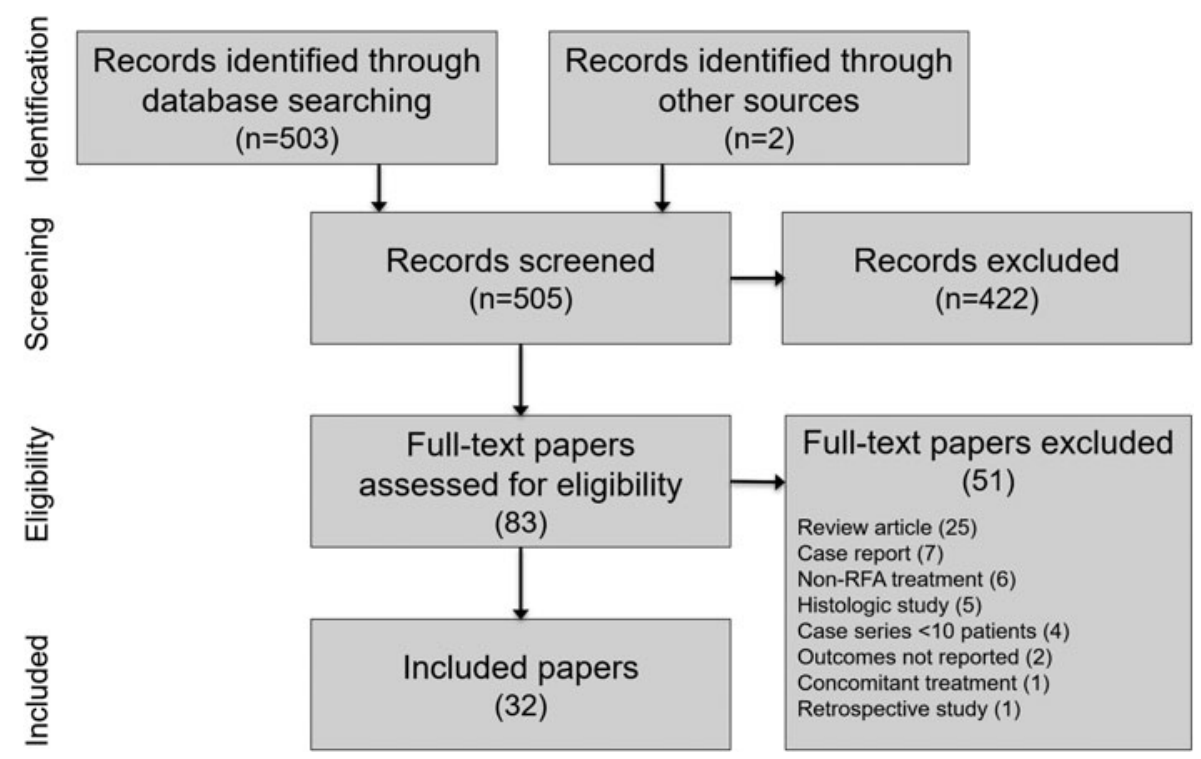

FIG. 1. PRISMA study flow diagram. PRISMA, Preferred Reporting Items for Systematic Reviews and Meta-analyses; RFA, radiofrequency ablation.

design characteristics are reported in Table 2. Among the 32 articles, 19 reported laparoscopic RFA, 8 reported transvaginal RFA, and 5 reported transcervical fibroid ablation (TFA). RFA was delivered using ultrasound guidance in $90 \%$ of the studies. Patient follow-up in each study ranged from in-hospital to 5.3 years (median 12 months).

Among the 20 prospective primary studies in this review (reported in 32 articles), study quality was rated as good or fair for 19 of 20 studies. The study design elements that were most frequently missing from published reports were interrupted time-series design ( 20 of 20 studies), blinded outcome assessors (20 of 20 studies), analyses that failed to adjust for attrition (19 of 20 studies), and no justification for sample size (15 of 20 studies) (Supplementary Table S3).

\section{Procedure and recovery results}

The weighted mean procedure time was 49 minutes $(95 \%$ CI: 41-56 minutes). Procedure time was significantly different among RFA delivery approaches (laparoscopic, 73 minutes; TFA, 44 minutes; transvaginal, 24 minutes), where all pairwise comparisons were $P \leq .002$. Time to discharge, time to normal activities, and time to return to work were reported inconsistently and, therefore, comparisons of RFA delivery approaches were reported descriptively only. The weighted mean time to discharge was 8.2 hours $(95 \% \mathrm{CI}$ : 6.3-10.0 hours), including 10.7 hours for laparoscopic RFA, 2.5 hours for TFA, and 2.5 hours for transvaginal RFA. The weighted mean time to normal activities was 5.2 days $(95 \%$ CI: 3.3-7.1 days), including 9.0 days for laparoscopic RFA, 3.3 days for TFA, and no studies for transvaginal RFA. The weighted mean return to work was 5.1 days (95\% CI: 3.7-6.5 days), including 6.5 days for laparoscopic RFA, 3.6 days for TFA, and no studies for transvaginal RFA (Table 3). Substantial heterogeneity among studies was observed for each of these outcomes, with $I^{2}$ ranging from $85 \%$ to $>99 \%$ (all $P<.001)$.

\section{Temporal trends in fibroid volume, fibroid-related quality of life, and reinterventions}

Following RFA, mean fibroid volume decreased by $47 \%$ at 3 months, $55 \%$ at 6 months, $66 \%$ at 12 months, and $71 \%$ at $>12$ months follow-up (Fig. 2). Low-to-moderate heterogeneity among studies was observed at each follow-up interval $\left(I^{2}\right.$ values of $54 \%[P=.02], 0 \%[P=.44], 43 \%[P=.07]$, and $0 \%[P=.42]$ at $3,6,12$, and $>12$ months, respectively). The percent change in fibroid volume 12 months after RFA was consistent across the range of treated fibroid volumes (Fig. 3). In metaregression that adjusted for differences in baseline fibroid volume, using laparoscopic RFA as the reference comparator, fibroid volume reduction was $4 \%$ greater with TFA $(P=.81)$ and $10 \%$ greater with transvaginal RFA $(P=.47)$ at 12 months.

Quality of life, where higher HRQL scores indicate better quality of life, improved relative to baseline by 30 points at 3 months, 37 points at 6 months, 39 points at 12 months, and 31 points at $>12$ months follow-up (all $P<.001$ versus baseline). Fibroid symptoms, where lower SSS scores indicate lower symptom severity, decreased by $29,36,42$, and 40 points relative to baseline over this same period (all $P<.001$ versus baseline) (Fig. 4). Considerable heterogeneity was evident at each follow-up interval for HRQL $\left(I^{2}\right.$ ranged from $86 \%$ to $99 \%$, all $\left.P<.001\right)$ and $\operatorname{SSS}\left(I^{2}\right.$ ranged from $46 \%$ to $99 \%$, all $P \leq .06$ ). The heterogeneity in HRQL (Fig. 5) and SSS (Fig. 6) changes was largely explained by the strong inverse association with the baseline value for that variable.

The cumulative rate of surgical reinterventions for fibroidrelated symptoms was $4.2 \%, 8.2 \%$, and $11.5 \%$ at annual follow-up intervals through 3 years (Fig. 7). The reintervention rate at 12 months was comparable among TFA (2.7\%), laparoscopic RFA (3.8\%), and transvaginal RFA (5.3\%), where $P \geq .52$ for all pairwise comparisons. The conclusions of this meta-analyses were unchanged among several sensitivity analyses (Supplementary Table S4). 


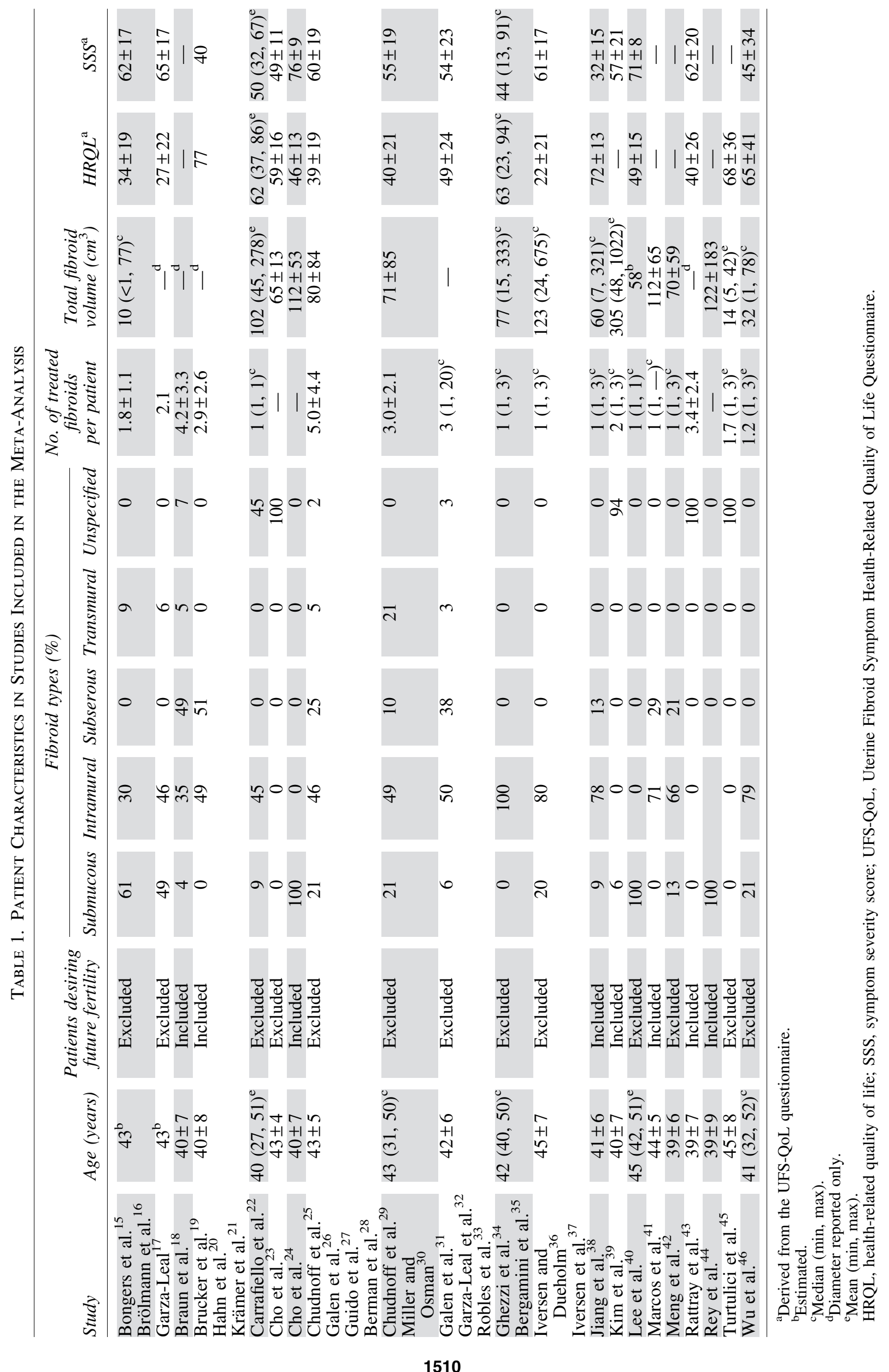




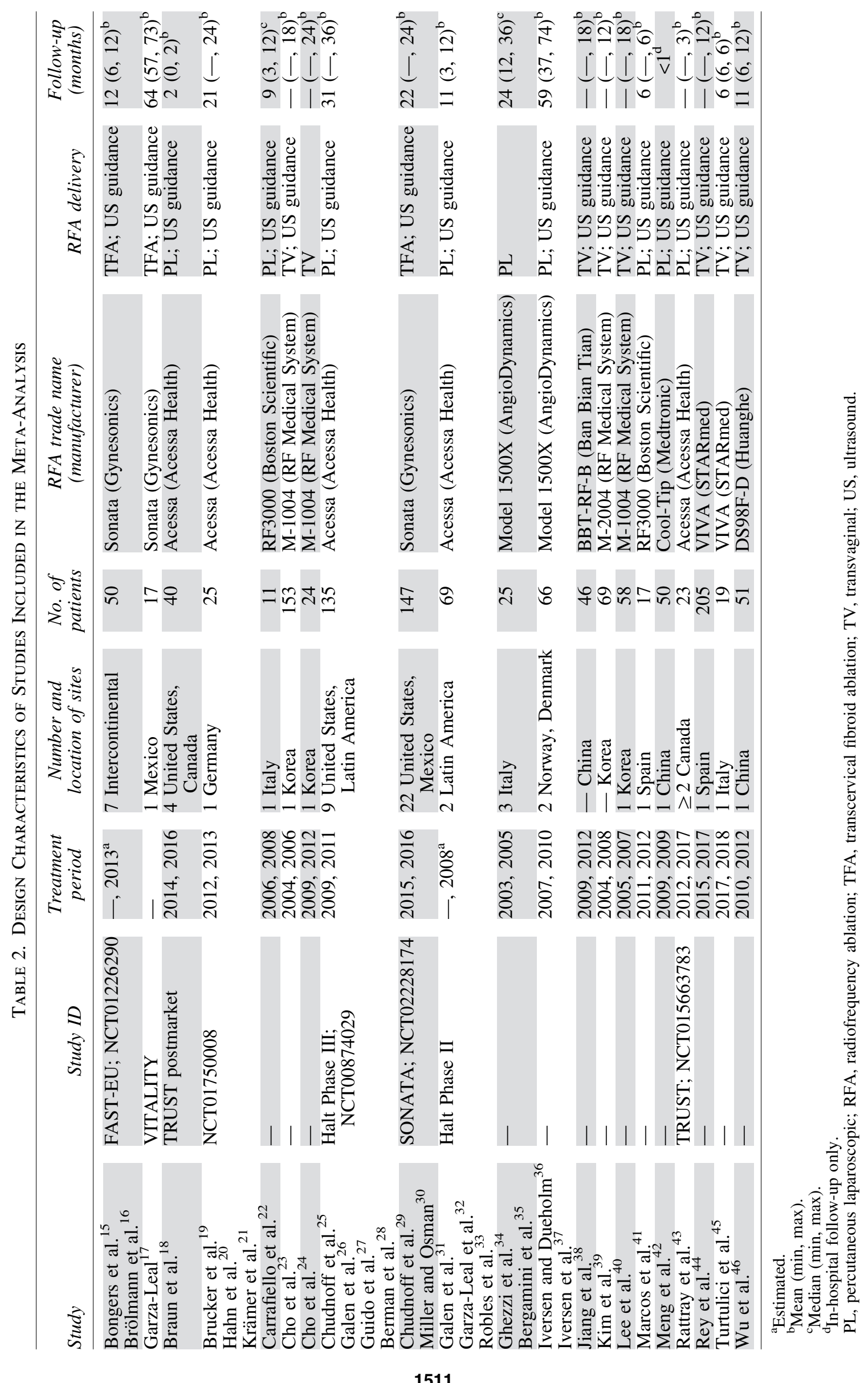


Table 3. Procedure and Recovery Results of Studies Included in the Meta-Analysis

\begin{tabular}{|c|c|c|c|c|}
\hline Study & $\begin{array}{c}\text { Procedure } \\
\text { time (minutes) }\end{array}$ & $\begin{array}{c}\text { Time to } \\
\text { discharge (hours) }\end{array}$ & $\begin{array}{c}\text { Time to return to } \\
\text { normal activities (days) }\end{array}$ & $\begin{array}{l}\text { Time to return } \\
\text { to work (days) }\end{array}$ \\
\hline $\begin{array}{l}\text { Bongers et al. }{ }^{15} \\
\text { Brölmann et al. }\end{array}$ & $39 \pm 23$ & - & $4.4 \pm 3.1$ & - \\
\hline Garza-Leal $^{17}$ & - & - & - & - \\
\hline Braun et al. ${ }^{18}$ & $114 \pm 60$ & $6.8 \pm 3.2$ & - & - \\
\hline $\begin{array}{l}\text { Brucker et al. }{ }^{19} \\
\text { Hahn et al. }{ }^{20} \\
\text { Krämer et }{ }^{21}\end{array}$ & $66 \pm 24$ & $10.0 \pm 5.5$ & $20.5(5,103)^{\mathrm{a}}$ & $10.0(2,86)^{\mathrm{a}}$ \\
\hline Carrafiello et al. 22 & $20(15,25)^{b}$ & - & - & - \\
\hline Cho et al. ${ }^{23}$ & $-(10,40)$ & - & - & - \\
\hline Cho et al. ${ }^{24}$ & - & - & - & - \\
\hline $\begin{array}{l}\text { Chudnoff et al. } \\
\text { Galen et al. }{ }^{26} \\
\text { Guido et al. } \\
\text { Berman et a }\end{array}$ & $126 \pm 60$ & - & $9.0(2,60)^{\mathrm{a}}$ & $5.0(0,29)^{\mathrm{a}}$ \\
\hline $\begin{array}{l}\text { Chudnoff et al. } \\
\text { Miller and Osman } \\
\text { 30 }\end{array}$ & $47 \pm 30$ & $2.5 \pm 1.2$ & $2.2 \pm 2.2$ & $3.6 \pm 2.6$ \\
\hline $\begin{array}{l}\text { Galen et al. } \\
\text { Garza-Leal et al. } \\
\text { Robles et al. }{ }^{33}\end{array}$ & $140(42,290)^{\mathrm{a}}$ & - & $4.5(1,11)^{b}$ & $4.0(2,10)^{\mathrm{a}}$ \\
\hline $\begin{array}{l}\text { Ghezzi et al. } \\
\text { Bergamini et al. }\end{array}$ & $25(20,45)^{\mathrm{a}}$ & $18^{\mathrm{c}}$ & - & - \\
\hline $\begin{array}{l}\text { Iversen and Dueholm } \\
\text { Iversen et al. }\end{array}$ & - & - & - & - \\
\hline Jiang et al. ${ }^{38}$ & $25(20,30)^{\mathrm{b}}$ & - & - & - \\
\hline Kim et al. ${ }^{39}$ & $18 \pm 5$ & - & - & - \\
\hline Lee et ll. $^{40}$ & - & - & - & - \\
\hline Marcos et al. ${ }^{41}$ & $36 \pm 11$ & $12.0(8,24)^{\mathrm{b}}$ & - & - \\
\hline Meng et al. ${ }^{42}$ & - & - & - & - \\
\hline Rattray et al. ${ }^{43}$ & $73 \pm 26$ & $6.7 \pm 3.0$ & - & $11.1 \pm 7.6$ \\
\hline Rey et $\mathrm{al}^{44}$ & $17(11,44)^{\mathrm{b}}$ & - & - & - \\
\hline Turtulici et al. ${ }^{45}$ & $28(16,43)^{\mathrm{b}}$ & - & - & - \\
\hline Wu et al. ${ }^{46}$ & $-(20,40)$ & $2.5^{\mathrm{c}}$ & - & - \\
\hline POOLED RESULT $^{d}$ & $49(41-56)$ & $8.2(6.3-10.0)$ & $5.2(3.3-7.1)$ & $5.1(3.7-6.5)$ \\
\hline Laparoscopic RFA & $73(56-91)$ & $10.7(5.9-15.5)$ & $9.0(3.8-14.1)$ & $6.5(3.8-9.2)$ \\
\hline Transvaginal RFA & $24(20-28)$ & $2.5(2.4-2.6)$ & - & - \\
\hline TFA & $44(36-51)$ & $2.5(2.3-2.7)$ & $3.3(1.1-5.4)$ & $3.6(3.1-4.1)$ \\
\hline
\end{tabular}

${ }^{\mathrm{a} M e d i a n}(\min , \max )$.

${ }^{\mathrm{b}}$ Mean (min, max).

${ }^{\mathrm{c}}$ Estimated value.

${ }^{\mathrm{d}}$ Pooled results derived from random effects meta-analysis and reported as weighted mean (95\% confidence interval).

RFA, radiofrequency ablation; TFA, transcervical fibroid ablation.

\section{Discussion}

RFA has been used with increasing frequency over the last decade to treat women with uterine fibroids who wish to preserve their uteri and possibly avoid more invasive surgery. Yet there is a paucity of comprehensive reviews regarding RFA of uterine fibroids that synthesize published evidence to help inform women and their gynecologists about typical acute and longer term results. In this systematic review and meta-analysis, the mean RFA procedure time was 49 minutes and performed on an outpatient basis in most cases. Patients returned to normal activities and to work in 5 days, on average, after RFA. We observed significant variability among studies for several outcomes, which was largely attributable to differences in baseline fibroid volume, quality of life, and RFA delivery approaches. Despite this variability, there was strong evidence of sustained fibroid volume reduction, significant improvements in HRQL and SSS, and favorable surgical reintervention rates following RFA.

Several systematic reviews have reported results of RFA for uterine fibroids. Lim et al. ${ }^{5}$ reported that laparoscopic

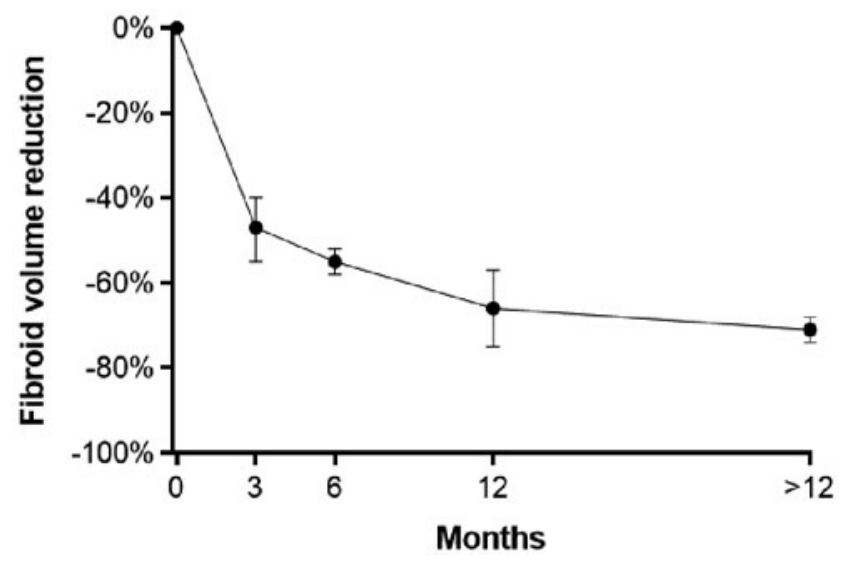

FIG. 2. Temporal trends in uterine fibroid volume following radiofrequency ablation of uterine fibroids. Plotted data are mean percent change from baseline and $95 \%$ confidence interval. Fibroid volumes at each follow-up interval were significantly smaller than baseline (all $P<.001$ ). Heterogeneity estimates were $I^{2}=54 \%(P=.02)$ at 3 months, $I^{2}=0 \%(P=.44)$ at 6 months, $I^{2}=43 \%(P=.07)$ at 12 months, and $I^{2}=0 \%$ $(P=.42)$ after 12 months. 


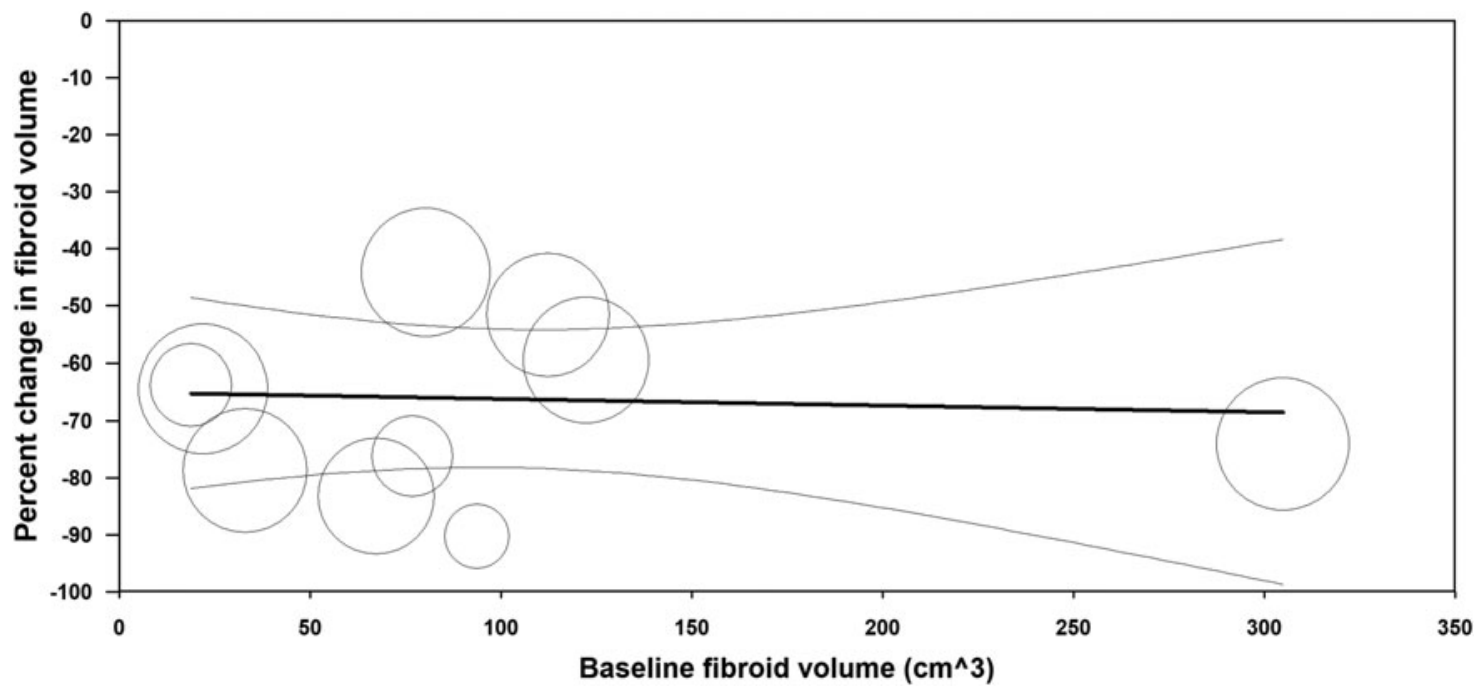

FIG. 3. Relationship between uterine fibroid volume at baseline with percent change in uterine volume following radiofrequency ablation. Plotted data are the metaregression line (dark line) and 95\% confidence interval (light lines), with results of individual studies denoted by circles, where circle size is proportional to the weighting of the study in the metaanalysis. Percentage of between-study variance explained by baseline fibroid volume $\left(R^{2}\right.$ analog $)=0 \%(P=.83)$.

RFA reduced uterine fibroid volume by $81 \mathrm{~cm}^{3}(P<.001)$, reduced SSS by 43 points $(P<.001)$, and improved HRQL by 38 points $(P<.001)$, with a reintervention rate of $2.7 \%$ over follow-up ranging from 9 to 36 months. More recently, Lin et al. ${ }^{6}$ performed a similar review that included the same RFA studies and ultimately reached the same conclusions.

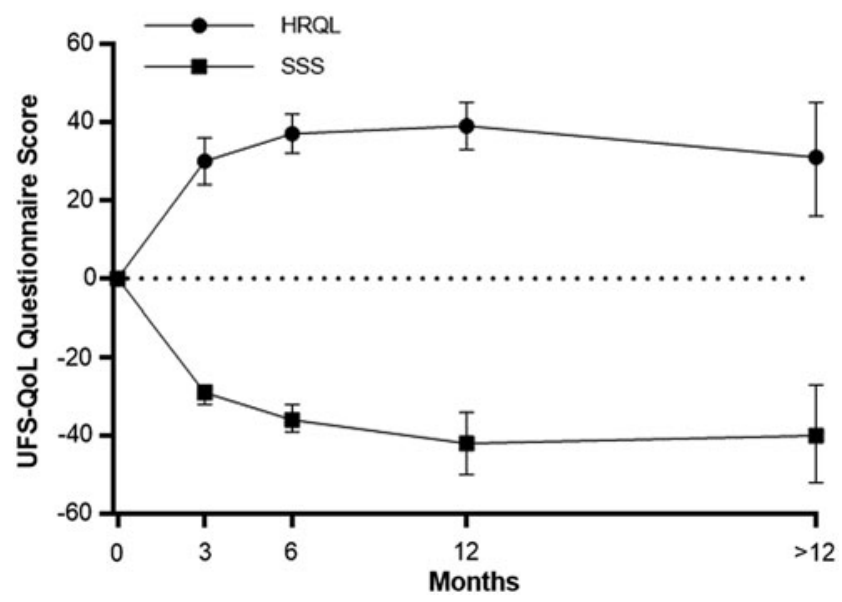

FIG. 4. Temporal trends in UFS-QoL subscores following radiofrequency ablation of uterine fibroids. Plotted data are mean absolute change from baseline and $95 \%$ confidence interval. HRQL values at each follow-up interval were significantly higher than baseline (all $P<.001$ ). SSS values at each follow-up interval were significantly lower than baseline (all $P<.001)$. Heterogeneity estimates for HRQL were $I^{2}=89 \%(P<.001)$ at 3 months, $I^{2}=86 \%(P<.001)$ at 6 months, $I^{2}=91 \%(P<.001)$ at 12 months, and $I^{2}=99 \%$ $(P<.001)$ after 12 months. Heterogeneity estimates for SSS were $I^{2}=46 \%(P=.06)$ at 3 months, $I^{2}=77 \%(P<.001)$ at 6 months, $I^{2}=96 \%(P<.001)$ at 12 months, and $I^{2}=99 \%$ $(P<.001)$ after 12 months. HRQL, health-related quality of life; SSS, symptom severity score; UFS-QoL, Uterine Fibroid Symptom Health-Related Quality of Life Questionnaire.
Sandberg et al. ${ }^{11}$ reported a reintervention rate of $0.3 \%$ at 1 year and $10.4 \%$ at 3 years, reductions in SSS of 37 points at 1 year, and increases in HRQL of 35 points at 1 year after laparoscopic RFA. Taheri et al. ${ }^{12}$ published a review comparing uterine artery embolization, various routes of RFA, and focused ultrasound and found that RFA provided a significantly greater percentage of fibroid volume reduction compared with the other treatments. No previous report has analyzed aggregate outcomes of transvaginal RFA or TFA studies. In this study, we report temporal trends in RFA outcomes, provide comparisons of outcomes by RFA delivery approach, and performed several sensitivity analyses to determine whether the meta-analysis conclusions were robust to various assumptions. Thus, the current review provides novel clinical evidence that arguably represents the most thorough meta-analysis results of RFA for treatment of uterine fibroids.

RFA was used to treat a wide variety of fibroid types and sizes in the included studies. Analysis of fibroid volume decreases in relation to baseline fibroid volumes suggests that RFA provides $\sim 65 \%$ reductions in fibroid volume across a broad range of fibroid sizes. Similarly, despite variation in baseline quality of life scores among studies, RFA provided significant improvements in SSS and HRQL at all follow-up intervals and across the entire range of preprocedural quality-of-life scores.

We also analyzed patient outcomes by RFA delivery approach, which revealed several important observations. First, TFA was associated with a brief mean procedure time, short mean length of stay, and, on average, a faster return to normal activities and work compared with laparoscopic RFA. Transvaginal RFA was also associated with short procedure time and length of stay, but no data related to time to return to normal activities or work were reported. It would be expected that the transvaginal and transcervical routes of RFA would provide similar outcomes, although in theory, TFA may be expected to be the safer of the two options given the need with transvaginal RFA to violate the uterine serosa with a charged 


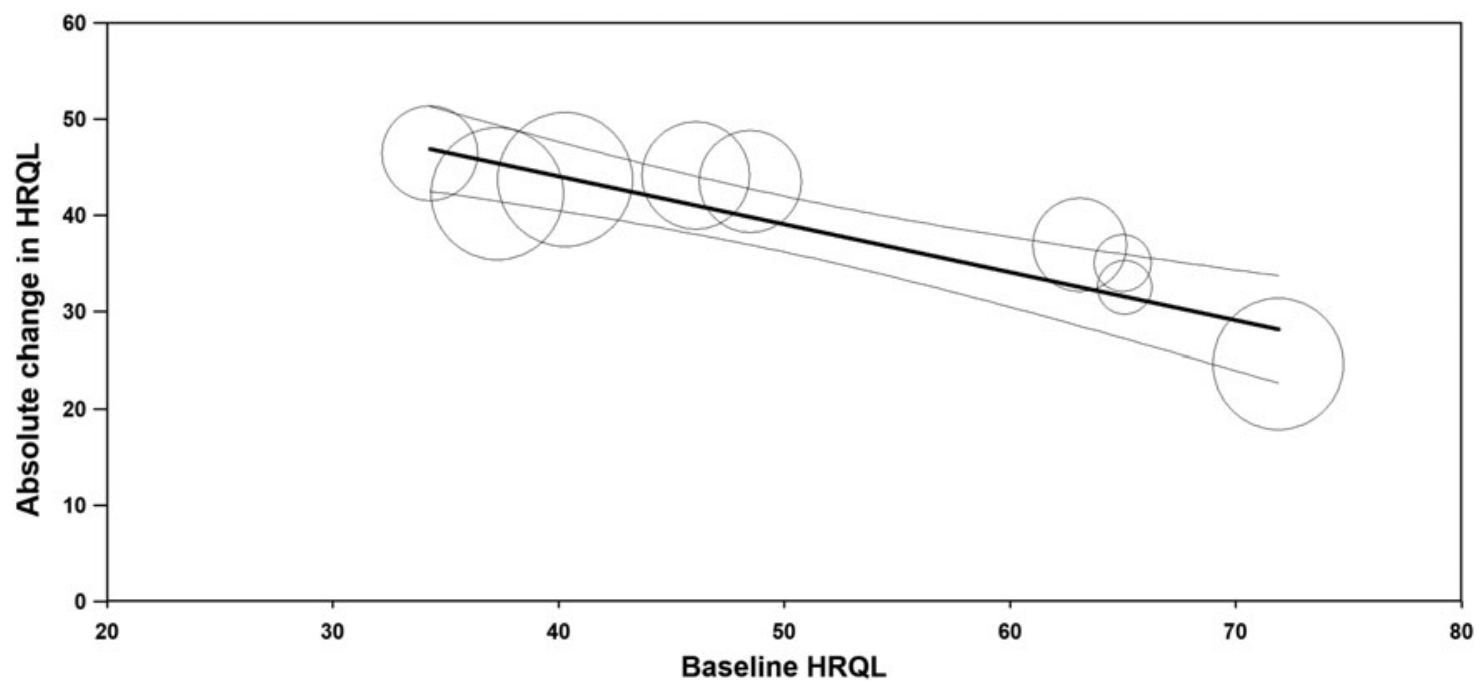

FIG. 5. Relationship between HRQL at baseline with HRQL change at 12 months following radiofrequency ablation. Plotted data are the metaregression line (dark line) and 95\% confidence interval (light lines), with results of individual studies denoted by circles, where circle size is proportional to the weighting of the study in the meta-analysis. Percentage of between-study variance explained by baseline HRQL $\left(R^{2}\right.$ analog $)=93 \%(P<.001)$. HRQL, healthrelated quality of life.

electrode or electrode array. Second, RFA delivery approaches were similarly effective in reducing fibroid volume and improving quality of life. Third, surgical reintervention rates for fibroid-related symptoms were favorable after RFA and did not significantly differ among RFA delivery approaches. Furthermore, the rate of reintervention at 3 years was $11.5 \%$ in the current review, which favorably compares with reported rates of $17 \%$ for uterine artery embolization, $21 \%$ for hysteroscopic myomectomy, $24 \%$ for endometrial ablation, and $11 \%$ for laparoscopic myomectomy over the same period. ${ }^{13}$
Main strengths of this review included adherence to PRISMA guidelines, excellent generalizability of results given the inclusion of almost 1300 patients, and robust conclusions that were unchanged in various sensitivity analyses. There are also several limitations pertaining to the quality of the included studies that may influence conclusions. First, there is less precision in the RFA results after 12 months of follow-up since fewer studies reported longer term data. Second, the number of included studies was insufficient to perform statistical comparisons among RFA delivery approaches for several outcomes. In these cases where we

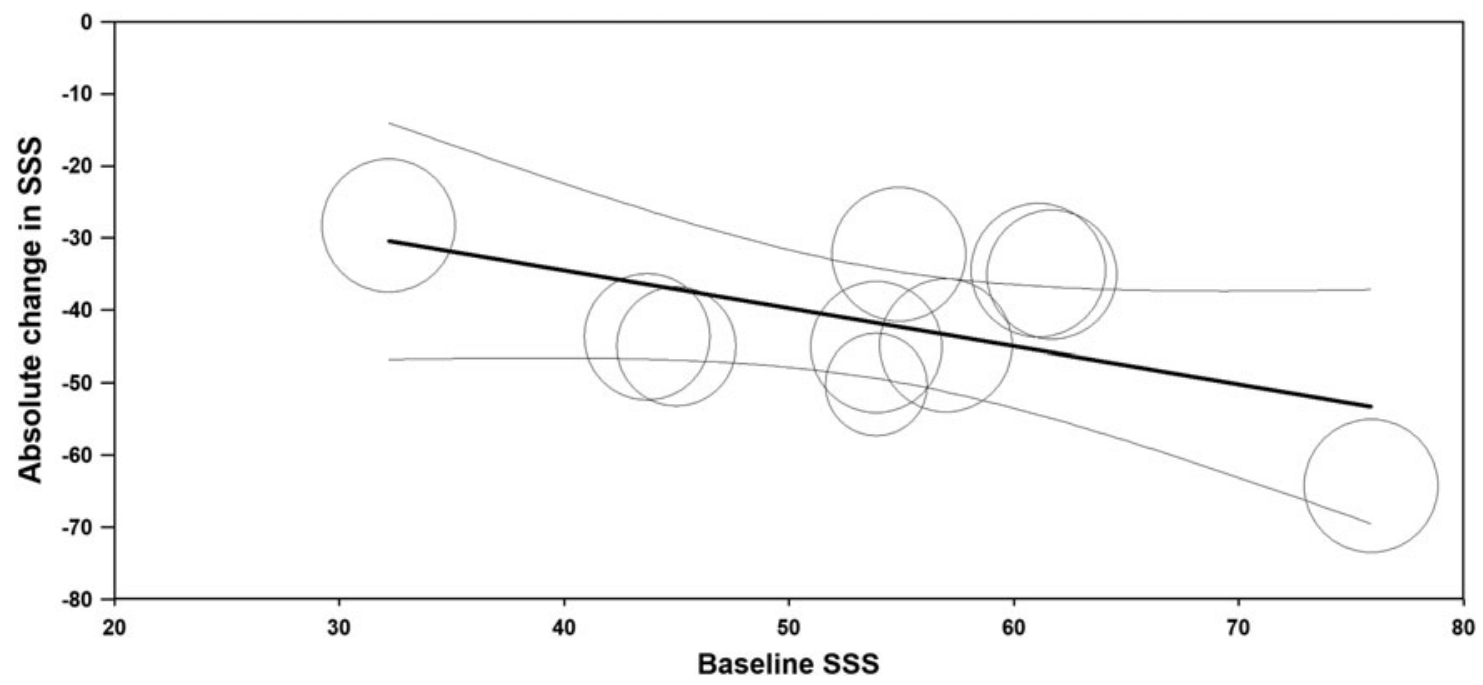

FIG. 6. Relationship between SSS at baseline with SSS change at 12 months following radiofrequency ablation. Plotted data are the metaregression line (dark line) and 95\% confidence interval (light lines), with results of individual studies denoted by circles, where circle size is proportional to the weighting of the study in the meta-analysis. Percentage of between-study variance explained by baseline SSS $\left(R^{2}\right.$ analog $)=44 \%(P=.05)$. SSS, symptom severity score. 


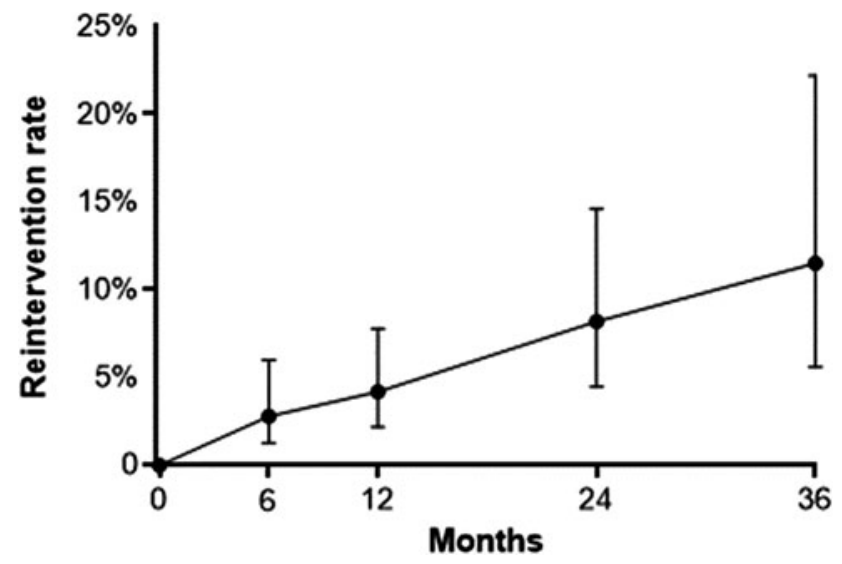

FIG. 7. Cumulative reintervention rate following radiofrequency ablation. Error bars are $95 \%$ confidence intervals.

reported the results descriptively, it is plausible that clinically important differences in patient outcomes existed among RFA delivery approaches that were not detectable in our meta-analysis due to insufficient statistical power. Importantly, since the comparative RFA outcomes reported in this study were derived from different studies and analyzed through metaregression, the post hoc results should be considered hypothesis generating only. Third, it is not possible to determine from this analysis whether RFA efficacy was influenced by fibroid type or volume due to concerns of aggregation bias whereby real associations observed at the patient level (e.g., fibroid volume in each patient) often do not agree with those observed at the study level (e.g., mean fibroid volume in each study). ${ }^{14}$ Finally, we planned to report the frequency of complications in this meta-analysis. Unfortunately, complication reporting was highly inconsistent and inadequate such that any attempts at reporting these data would have led to inaccurate and misleading results. For example, most articles provided no criteria or definitions regarding complication reporting. Furthermore, several articles simply reported that no complications occurred without any further commentary. Regardless, no serious procedural complications such as death or iatrogenic injury to the bowel, bladder, or ureter were reported in any study. Authors of future RFA studies are encouraged to provide detailed definitions of complications and a complete listing of reported complications during follow-up, with further specification of event seriousness and relationship to the RFA procedure. Lastly, RFA was utilized across a broad range of fibroid types and volumes, suggesting that this therapy is appropriate for most women with symptomatic uterine fibroids.

\section{Conclusion}

RFA of uterine fibroids significantly reduces fibroid volume, provides significant improvements in fibroid-related quality of life, and is associated with favorable reintervention rates.

\section{Acknowledgments}

The authors thank David Fay, PhD for assistance with literature review and data extraction, and David Toub, MD, MBA and Taraneh G. Farazi, PhD for contributing feedback to this article.

\section{Disclosure Statement}

L.D.B. reports principal investigator grant support from Bayer, scientific advisory boards for AbbVie, Bayer, Allergan, and PCORI, and royalties from Elsevier and Wolter Kluwer, all unrelated to the submitted work. R.P.P. reports consultancy with Ethicon Endo, Medtronic, Cooper Surgical, and Olympus, all unrelated to the submitted work. L.E.M. reports consultancy with Gynesonics, Inc., related to the submitted work.

\section{Funding Information}

Gynesonics, Inc., provided funding for this study.

\section{Supplementary Material}

Supplementary Table S1

Supplementary Table S2

Supplementary Table S3

Supplementary Table S4

\section{References}

1. Baird DD, Dunson DB, Hill MC, Cousins D, Schectman JM. High cumulative incidence of uterine leiomyoma in black and white women: Ultrasound evidence. Am J Obstet Gynecol 2003;188:100-107.

2. Zimmermann A, Bernuit D, Gerlinger C, Schaefers M, Geppert K. Prevalence, symptoms and management of uterine fibroids: An international internet-based survey of 21,746 women. BMC Womens Health 2012;12:6.

3. Borah BJ, Nicholson WK, Bradley L, Stewart EA. The impact of uterine leiomyomas: A national survey of affected women. Am J Obstet Gynecol 2013;209:319 e1e19 e20.

4. Luo X, Shen Y, Song WX, Chen PW, Xie XM, Wang XY. Pathologic evaluation of uterine leiomyoma treated with radiofrequency ablation. Int J Gynaecol Obstet 2007;99:9-13.

5. Lim KJ, Yoon DY, Kim JH, et al. Percutaneous radiofrequency ablation for symptomatic uterine leiomyomas: A systematic review and meta-analysis. Clin Exp Obstet Gynecol 2016;43:643-649.

6. Lin L, Ma H, Wang J, et al. Quality of life, adverse events, and reintervention outcomes after laparoscopic radiofrequency ablation for symptomatic uterine fibroids: A metaanalysis. J Minim Invasive Gynecol 2019;26:409-416.

7. Liberati A, Altman DG, Tetzlaff J, et al. The PRISMA statement for reporting systematic reviews and metaanalyses of studies that evaluate health care interventions: Explanation and elaboration. Ann Intern Med 2009;151: W65-W94.

8. Spies JB, Coyne K, Guaou Guaou N, Boyle D, SkyrnarzMurphy K, Gonzalves SM. The UFS-QOL, a new disease-specific symptom and health-related quality of life questionnaire for leiomyomata. Obstet Gynecol 2002;99: 290-300.

9. National Institute of Health. Quality Assessment Tool for Before-After (Pre-Post) Studies With No Control Group. Available at: https://www.nhlbi.nih.gov/health-topics/studyquality-assessment-tools (last accessed June 2, 2019).

10. Higgins JP, Thompson SG, Deeks JJ, Altman DG. Measuring inconsistency in meta-analyses. BMJ 2003;327:557-560.

11. Sandberg EM, Tummers F, Cohen SL, Van Den Haak L, Dekkers OM, Jansen FW. Reintervention risk and quality of life outcomes after uterine-sparing interventions for fi- 
broids: A systematic review and meta-analysis. Fertil Steril 2018;109:698-707 e1.

12. Taheri M, Galo L, Potts C, Sakhel K, Quinn SD. Nonresective treatments for uterine fibroids: A systematic review of uterine and fibroid volume reductions. Int $\mathrm{J}$ Hyperthermia 2019;36:295-301.

13. Davis MR, Soliman AM, Castelli-Haley J, Snabes MC, Surrey ES. Reintervention rates after myomectomy, endometrial ablation, and uterine artery embolization for patients with uterine fibroids. J Womens Health (Larchmt) 2018;27:1204-1214.

14. Cooper H, Patall EA. The relative benefits of meta-analysis conducted with individual participant data versus aggregated data. Psychol Methods 2009;14:165-176.

15. Bongers M, Brölmann H, Gupta J, Garza-Leal JG, Toub D. Transcervical, intrauterine ultrasound-guided radiofrequency ablation of uterine fibroids with the VizAblate(R) System: Three- and six-month endpoint results from the FAST-EU study. Gynecol Surg 2015;12:61-70.

16. Brölmann H, Bongers M, Garza-Leal JG, et al. The FASTEU trial: 12-month clinical outcomes of women after intrauterine sonography-guided transcervical radiofrequency ablation of uterine fibroids. Gynecol Surg 2016; 13:27-35.

17. Garza-Leal JG. Long-term clinical outcomes of transcervical radiofrequency ablation of uterine fibroids: The VITALITY study. J Gynecol Surg 2019;35:19-23.

18. Braun KM, Sheridan M, Latif EZ, et al. Surgeons' early experience with the Acessa procedure: Gaining proficiency with new technology. Int J Womens Health 2016;8:669675.

19. Brucker SY, Hahn M, Kraemer D, Taran FA, Isaacson KB, Krämer B. Laparoscopic radiofrequency volumetric thermal ablation of fibroids versus laparoscopic myomectomy. Int J Gynaecol Obstet 2014;125:261-265.

20. Hahn M, Brucker S, Kraemer D, et al. Radiofrequency volumetric thermal ablation of fibroids and laparoscopic myomectomy: Long-term follow-up from a randomized trial. Geburtshilfe Frauenheilkd 2015;75:442-449.

21. Krämer B, Hahn M, Taran FA, Kraemer D, Isaacson KB, Brucker SY. Interim analysis of a randomized controlled trial comparing laparoscopic radiofrequency volumetric thermal ablation of uterine fibroids with laparoscopic myomectomy. Int J Gynaecol Obstet 2016;133:206-211.

22. Carrafiello G, Recaldini C, Fontana F, et al. Ultrasoundguided radiofrequency thermal ablation of uterine fibroids: Medium-term follow-up. Cardiovasc Intervent Radiol 2010;33:113-119.

23. Cho HH, Kim JH, Kim MR. Transvaginal radiofrequency thermal ablation: A day-care approach to symptomatic uterine myomas. Aust N Z J Obstet Gynaecol 2008;48: 296-301.

24. Cho HH, Kim MR, Kim JH. Outpatient multimodality management of large submucosal myomas using transvaginal radiofrequency myolysis. J Minim Invasive Gynecol 2014;21:1049-1054.

25. Chudnoff SG, Berman JM, Levine DJ, Harris M, Guido RS, Banks E. Outpatient procedure for the treatment and relief of symptomatic uterine myomas. Obstet Gynecol 2013;121: 1075-1082.

26. Galen DI, Isaacson KB, Lee BB. Does menstrual bleeding decrease after ablation of intramural myomas? A retrospective study. J Minim Invasive Gynecol 2013;20: 830-835.

27. Guido RS, Macer JA, Abbott K, Falls JL, Tilley IB, Chudnoff SG. Radiofrequency volumetric thermal ablation of fibroids: A prospective, clinical analysis of two years' outcome from the Halt trial. Health Qual Life Outcomes 2013;11:139.

28. Berman JM, Guido RS, Garza-Leal JG, et al. Three-year outcome of the Halt trial: A prospective analysis of radiofrequency volumetric thermal ablation of myomas. J Minim Invasive Gynecol 2014;21:767-774.

29. Chudnoff S, Guido R, Roy K, Levine D, Mihalov L, GarzaLeal JG. Ultrasound-guided transcervical ablation of uterine leiomyomata. Obstet Gynecol 2019;133:13-22.

30. Miller CE, Osman K. Transcervical radiofrequency ablation of symptomatic uterine fibroids: 2-year results of the SONATA pivotal trial. J Gynecol Surg 2019 [Epub ahead of print]; DOI: 10.1089/gyn.2019.0012.

31. Galen DI, Pemueller RR, Leal JG, Abbott KR, Falls JL, Macer J. Laparoscopic radiofrequency fibroid ablation: Phase II and phase III results. JSLS 2014;18:182-190.

32. Garza-Leal JG, Hernandez Leon I, Castillo Saenz L, Lee BB. Laparoscopic ultrasound-guided radiofrequency volumetric thermal ablation of symptomatic uterine leiomyomas: Feasibility study using the Halt 2000 Ablation System. J Minim Invasive Gynecol 2011;18:364-371.

33. Robles R, Aguirre VA, Argueta AI, Guerrero MR. Laparoscopic radiofrequency volumetric thermal ablation of uterine myomas with 12 months of follow-up. Int J Gynaecol Obstet 2013;120:65-69.

34. Ghezzi F, Cromi A, Bergamini V, Scarperi S, Bolis P, Franchi M. Midterm outcome of radiofrequency thermal ablation for symptomatic uterine myomas. Surg Endosc 2007;21:2081-2085.

35. Bergamini V, Ghezzi F, Cromi A, et al. Laparoscopic radiofrequency thermal ablation: A new approach to symptomatic uterine myomas. Am J Obstet Gynecol 2005; 192:768-773.

36. Iversen H, Dueholm M. Radiofrequency thermal ablation for uterine myomas: Long-term clinical outcomes and reinterventions. J Minim Invasive Gynecol 2017;24: 1020-1028.

37. Iversen H, Lenz S, Dueholm M. Ultrasound-guided radiofrequency ablation of symptomatic uterine fibroids: Shortterm evaluation of effect of treatment on quality of life and symptom severity. Ultrasound Obstet Gynecol 2012;40: $445-451$

38. Jiang X, Thapa A, Lu J, Bhujohory VS, Liu Y, Qiao S. Ultrasound-guided transvaginal radiofrequency myolysis for symptomatic uterine myomas. Eur J Obstet Gynecol Reprod Biol 2014;177:38-43.

39. Kim CH, Kim SR, Lee HA, Kim SH, Chae HD, Kang BM. Transvaginal ultrasound-guided radiofrequency myolysis for uterine myomas. Hum Reprod 2011;26:559-563.

40. Lee Y, Cho HH, Kim JH, et al. Radiofrequency thermal ablation of submucosal leiomyoma: A preliminary report on health, symptom, and quality of life outcomes. J Gynecol Surg 2010;26:227-231.

41. Marcos RG, Monleón J, Martínez-Varea A, et al. Percutaneous ultrasound-guided radiofrequency thermal ablaiton for treatment of uterine fibroids. Open J Obstet Gynecol 2014;4:716-724. 
42. Meng X, He G, Zhang J, et al. A comparative study of fibroid ablation rates using radio frequency or highintensity focused ultrasound. Cardiovasc Intervent Radiol 2010;33:794-799.

43. Rattray DD, Weins L, Regush LC, Bowen JM, O'reilly D, Thiel JA. Clinical outcomes and health care utilization pre- and post-laparoscopic radiofrequency ablation of symptomatic fibroids and laparoscopic myomectomy: A randomized trial of uterine-sparing techniques (TRUST) in Canada. Clinicoecon Outcomes Res 2018;10:201-212.

44. Rey VE, Labrador R, Falcon M, Garcia-Benitez JL. Transvaginal radiofrequency ablation of myomas: Technique, outcomes, and complications. J Laparoendosc Adv Surg Tech A 2019;29:24-28.

45. Turtulici G, Orlandi D, Dedone G, et al. Ultrasound-guided transvaginal radiofrequency ablation of uterine fibroids assisted by virtual needle tracking system: A preliminary study. Int J Hyperthermia 2019;35:97-104.

46. Wu XJ, Guo Q, Cao BS, et al. Uterine leiomyomas: Safety and efficacy of US-guided suprapubic transvaginal radiofrequency ablation at 1-year follow-up. Radiology 2016; 279:952-960.

Address correspondence to: Larry E. Miller, PhD Miller Scientific Consulting, Inc. 1854 Hendersonville Road, \#231 Asheville, NC 28803

E-mail: larry@millerscientific.com 